PROCEEDINGS OF THE

AMERICAN MATHEMATICAL SOCIETY

Volume 137, Number 8, August 2009, Pages 2813-2815

S 0002-9939(09)09856-6

Article electronically published on April 2, 2009

\title{
ERRATUM TO: COMPACTNESS PROPERTIES FOR OPERATORS DOMINATED BY AM-COMPACT OPERATORS
}

\author{
BELMESNAOUI AQZZOUZ AND AZIZ ELBOUR
}

(Communicated by Nigel J. Kalton)

In [3, Theorem 2.10] it was proposed to characterize Banach lattices such that operators dominated by AM-compact operators are AM-compact. But there was an error in the proof of the above-mentioned theorem. The purpose of this erratum is to give a new and correct proof of Theorem 2.10 of $[3$. Let us recall that if $E$ is a Banach lattice, $E^{\prime}$ is its topological dual and $\varphi \in E^{\prime}$, the null ideal of $\varphi$ is defined by $N_{\varphi}=\{x \in E:|\varphi|(|x|)=0\}$ and the carrier $C_{\varphi}$ of $\varphi \in E^{\prime}$ is defined by $C_{\varphi}=\left(N_{\varphi}\right)^{d}=\left\{u \in E:|u| \wedge|v|=0\right.$ for all $\left.v \in N_{\varphi}\right\}$.

To give our proof, we will need the following lemma:

Lemma 1. Let $E$ be a Banach lattice. If the norm of $E$ is not order continuous, then there exist $y \in E^{+}$and a disjoint sequence $\left(y_{n}\right) \subset[0, y]$ such that $\left\|y_{n}\right\|=1$ for all $n$. Moreover, there exists a positive disjoint sequence $\left(g_{n}\right)$ of $E^{\prime}$ with $\left\|g_{n}\right\| \leq 1$ such that $g_{n}\left(y_{n}\right)=1$ for all $n$ and $g_{n}\left(y_{m}\right)=0$ for $n \neq m$.

Proof. If the norm of $E$ is not order continuous, then Theorem 4.14 of 2 implies the existence of some $u \in E^{+}$and a disjoint sequence $\left(u_{n}\right)$ in $[0, u]$ which does not converge to zero in norm. By choosing a subsequence we may suppose that $\left\|u_{n}\right\|>\varepsilon$ for all $n$ and some $\varepsilon>0$. If we take $y_{n}=\frac{u_{n}}{\left\|u_{n}\right\|}$ and $y=\frac{u}{\varepsilon}$, we obtain a disjoint sequence $\left(y_{n}\right)$ in $[0, y]$ satisfying $\left\|y_{n}\right\|=1$ for all $n$.

On the other hand, by Theorem 39.3 of [6], for each $n$ there exists $f_{n} \in\left(E^{\prime}\right)^{+}$ such that $\left\|f_{n}\right\|=1$ and $f_{n}\left(y_{n}\right)=\left\|y_{n}\right\|=1$. Under the natural embedding of $E$ into its topological bidual $E^{\prime \prime}$, the space $E$ becomes a sublattice of $\left(E^{\prime}\right)_{n}^{\prime}$. This implies that $\left(y_{n}\right)$ is a disjoint sequence of positive order continuous functionals on $E^{\prime}$. Now, it follows from Nakano's Theorem [2, Theorem 1.67] that the carriers $C_{y_{n}}$ are mutually disjoint bands in $E^{\prime}$. If $g_{n}$ is the projection of $f_{n}$ onto $C_{y_{n}}$, then it is easy to verify that the sequence $\left(g_{n}\right)$ satisfies the desired properties.

Also, we shall need the following characterisation, which follows from Theorem 3.27 of [2].

Lemma 2. Let $E$ be a Banach lattice and $X$ a Banach space, and let $T: E \rightarrow X$ be an operator. Then $T$ is AM-compact if and only if $T^{\prime}\left(B_{X^{\prime}}\right)$ is precompact for the topology $|\sigma|\left(E^{\prime}, E\right)$, where $B_{X^{\prime}}$ is the closed unit ball of $X^{\prime}$.

Received by the editors July 25, 2008, and, in revised form, December 14, 2008.

2000 Mathematics Subject Classification. Primary 46A40, 46B40; Secondary 46B42.

Key words and phrases. AM-compact operator, order continuous norm, discrete Banach lattice.

(C)2009 American Mathematical Society Reverts to public domain 28 years from publication 
Let $E$ be a Banach lattice and let $u \in E^{+}$. Then the order ideal $E_{u}$ generated by $u$ with the norm $\|y\|_{\infty}=\inf \{\lambda>0:|y| \leq \lambda$. $u\}$ is an AM-space having $u$ as a unit and $[-u, u]$ as a closed unit ball, and the embedding $i_{u}:\left(E_{u},\|\cdot\|_{\infty}\right) \rightarrow E$ is continuous. Moreover, for every $f \in E^{\prime}$ we have $f \circ i_{u} \in\left(E_{u}\right)^{\prime}$ and

$$
\begin{aligned}
\left\|f \circ i_{u}\right\|_{\left(E_{u}\right)^{\prime}} & =\sup \left\{\left|\left(f \circ i_{u}\right)(y)\right|: y \in[-u, u]\right\} \\
& =\sup \{|f(y)|:|y| \leq u\} \\
& =|f|(u) .
\end{aligned}
$$

Now we are in a position to give the correct proof of Theorem 2.10 of 3 .

Theorem 1. Let $E$ and $F$ be two Banach lattices. Then the following statements are equivalent:

(1) For all operators $S, T: E \rightarrow F$ such that $0 \leq S \leq T$ and $T$ is $A M$-compact, the operator $S$ is AM-compact.

(2) One of the following conditions holds:

(a) the norm of $F$ is order continuous;

(b) $E^{\prime}$ is discrete.

Proof. $(2 . a) \Rightarrow(1)$ This is just a result of Fremlin (see [5, Proposition 3.7.2] for the proof).

$(2 . b) \Rightarrow(1)$ By Lemma 2, it suffices to show that $S^{\prime}\left(B_{F^{\prime}}\right)$ is precompact for $|\sigma|\left(E^{\prime}, E\right)$. Let $V$ be a solid neighborhood of zero for $|\sigma|\left(E^{\prime}, E\right)$. Since $T$ is AMcompact, it follows from Lemma 2 that $T^{\prime}\left(B_{F^{\prime}}\right)$ is precompact for $|\sigma|\left(E^{\prime}, E\right)$. Then there exists a finite subset $K$ of $E^{\prime}$ such that $T^{\prime}\left(B_{F^{\prime}}\right) \subset K+V$. Choose $0 \leq f \in E^{\prime}$ such that $K \subset[-f, f]$ and note that

$$
\left|S^{\prime}(g)\right| \leq S^{\prime}(|g|) \leq T^{\prime}(|g|) \in[-f, f]+V \quad \forall g \in B_{F^{\prime}} .
$$

Then

$$
S^{\prime}\left(B_{F^{\prime}}\right) \subset[-f, f]+V .
$$

Since $E^{\prime}$ is discrete and order complete and the topology $|\sigma|\left(E^{\prime}, E\right)$ is Lebesgue, it follows from Corollary 6.57 of [1] that $[-f, f]$ is compact for $|\sigma|\left(E^{\prime}, E\right)$. Finally, by $(*)$ we see that $S^{\prime}\left(B_{F^{\prime}}\right)$ is precompact for $|\sigma|\left(E^{\prime}, E\right)$.

$(1) \Rightarrow(2)$ Assume by way of contradiction that the condititons $(a)$ and $(b)$ fail. To finish the proof, we have to construct two operators $S, T: E \rightarrow F$ such that $T$ is AM-compact, $S$ is not AM-compact and $0 \leq S \leq T$.

Since $F$ does not have an order continuous norm, it follows from Lemma 1 that there exists $y \in F^{+}$and there is a disjoint sequence $\left(y_{n}\right) \subset[0, y]$ such that $\left\|y_{n}\right\|=1$ for each $n$ and there exists a positive disjoint sequence $\left(g_{n}\right)$ of $E^{\prime}$ with $\left\|g_{n}\right\| \leq 1$ such that $g_{n}\left(y_{n}\right)=1$ for all $n$ and $g_{n}\left(y_{m}\right)=0$ for $n \neq m$. $\quad(* *)$

On the other hand, as $E^{\prime}$ is not discrete, Theorem 3.1 of 4 implies the existence of a sequence $\left(f_{n}\right) \subset E^{\prime}$ such that $f_{n} \rightarrow 0$ for $\sigma\left(E^{\prime}, E\right)$ as $n \rightarrow \infty$ and $\left|f_{n}\right|=f>0$ for all $n$ and some $f \in E^{\prime}$.

Now, we consider the operators $S, T: E \rightarrow F$ defined by

$$
S(x)=\left(\sum_{n=1}^{\infty} f_{n}(x) y_{n}\right)+f(x) y \quad \text { and } \quad T(x)=2 f(x) y \quad \forall x \in E .
$$

The sum in the definition of $S$ is norm convergent for each $x \in E$, because $f_{n}(x) \rightarrow 0$ and the sequence $\left(y_{n}\right)$ is disjoint and order bounded. 
Clearly, $0 \leq S \leq T$ holds. (In fact, for each $x \in E^{+}$and each $n \geq 1$, we have

$$
\left|\sum_{k=1}^{n} f_{k}(x) y_{k}\right| \leq \sum_{k=1}^{n} f(x) y_{k} \leq f(x) y .
$$

Then $\left|\sum_{n=1}^{\infty} f_{n}(x) y_{n}\right| \leq f(x) y$ for each $x \in E^{+}$. Hence $0 \leq S(x) \leq T(x)$ for each $x \in E^{+}$.) Also, it is clear that $T$ is compact (it has rank one) and hence $T$ is AM-compact.

To end the proof, we need to prove that $S$ is not AM-compact. Choose $u \in E^{+}$ such that $f(u)>0$, and note that $\left(f_{n} \circ i_{u}\right)_{n}$ has no norm convergent subsequence in $\left(E_{u}\right)^{\prime}$. In fact, for each $y \in E_{u}$ we have $f_{n} \circ i_{u}(y)=f_{n}(y) \rightarrow 0$ as $n \rightarrow \infty$. Then $f_{n} \circ i_{u} \rightarrow 0$ for $\sigma\left(\left(E_{u}\right)^{\prime}, E_{u}\right)$. As $\left\|f_{n} \circ i_{u}\right\|_{\left(E_{u}\right)^{\prime}}=\left|f_{n}\right|(u)=f(u)>0$ for all $n$, we conclude that $\left(f_{n} \circ i_{u}\right)_{n}$ has no norm convergent subsequence in $\left(E_{u}\right)^{\prime}$.

If $S$ is AM-compact, then $S \circ i_{u}: E_{u} \rightarrow E \rightarrow F$ is compact and so is $\left(S \circ i_{u}\right)^{\prime}$. We have $\left(S \circ i_{u}\right)^{\prime}(g)=\left(\sum_{n=1}^{\infty} g\left(y_{n}\right) .\left(f_{n} \circ i_{u}\right)\right)+g(y) .\left(f \circ i_{u}\right)$ for all $g \in F^{\prime}$. Then, by $\left(^{* *}\right),\left(S \circ i_{u}\right)^{\prime}\left(g_{k}\right)=\left(f_{k} \circ i_{u}\right)+g_{k}(y) \cdot\left(f \circ i_{u}\right)$ for all $k$. Hence $\left(\left(S \circ i_{u}\right)^{\prime}\left(g_{k}\right)\right)_{k}$ has a norm convergent subsequence in $\left(E_{u}\right)^{\prime}$. Since $\left(g_{k}(y)\right)_{k} \subset[-\|y\|,\|y\|] \subset \mathbb{R}$ has a convergent subsequence (because it is a bounded sequence in $\mathbb{R}$ ), we conclude that $\left(f_{k} \circ i_{u}\right)_{k}$ has a convergent subsequence in $\left(E_{u}\right)^{\prime}$. This is a contradiction, and then $S$ is not AM-compact.

\section{REFERENCES}

[1] Aliprantis, C.D. and Burkinshaw, O., Locally solid Riesz spaces with applications to economics, volume 105 of Mathematical Surveys and Monographs. American Mathematical Society, Providence, RI, second edition, 2003. MR2011364 (2005b:46010)

[2] Aliprantis, C.D. and Burkinshaw, O., Positive operators. Reprint of the 1985 original. Springer, Dordrecht, 2006. MR2262133

[3] Aqzzouz, B., Nouira, R. and Zraoula, L., Compactness properties of operators dominated by AM-compact operators. Proc. Amer. Math. Soc. 135 (2007) 1151-1157. MR2262919 (2007j:47073)

[4] Chen, Z. L. and Wickstead, A. W., Some applications of Rademacher sequences in Banach lattices. Positivity 2 (1998), no. 2, 171-191. MR.1656870 (2000a:46028)

[5] Meyer-Nieberg. P., Banach lattices. Universitext. Springer-Verlag, Berlin, 1991. MR.1128093 (93f:46025)

[6] Zaanen, A.C., Introduction to operator theory in Riesz spaces. Springer-Verlag, Berlin, 1997. MR.1631533 (2000c:47074)

Département d’Économie, Faculté des Sciences Économiques, Juridiques et Sociales, Université Mohammed V-Souissi, B.P. 5295, Sala Eljadida, Morocco

E-mail address: baqzzouz@hotmail.com

Équipe d’Analyse Fonctionnelle, Département de Mathématiques, Faculté des Sciences, Université Ibn Tofall, B.P. 133, Kénitra, Morocco

E-mail address: azizelbour@hotmail.com 